factors which impact PFI in epithelial ovarian cancer in Tunisia.

Methods We conducted a retrospective monocentric study including 60 Tunisian patients with relapsed epithelial ovarian cancer between October 2012 and December 2018. Clinico- pathological data, treatment and survival data were collected from medical records. Predictive factors were identified by logistic regression following the Cox regression model.

Results The median age at first diagnosis was 59 years [2885 years]. Relapsed ovarian cancers were platinum-sensitive in $65 \%(\mathrm{n}=39)$ and resistant in $35 \%(\mathrm{n}=21)$ of cases. Overall 2-year survival was $87 \%$ in the sensitive relapse group versus $11 \%$ in the resistant group $(\mathrm{p}<0.001)$. Age, Body mass index, histologic type, grade and timing of initial surgery didn't impact significantly the interval of relapse. However, short platinum-free interval $(<6$ months $)$ was correlated with : advanced stage at diagnosis (IIA to IV) (Odds ratio, OR:1.67, 95\% Confidence interval, CI [1.342.09]) and incomplete staging at the initial surgery (OR: 3.4, 95\% CI [1.07-10.84].

Conclusions The interval of relapse is correlated with the extension of the disease and/or with the quality of surgery. Patients should be referred to expert centers.

\section{EPV214/\#506 OVERALL SURVIVAL PROGNOSTIC FACTORS IN RECURRENT EPITHELIAL OVARIAN CANCER IN TUNISIA}

${ }^{1} \mathrm{Y}$ Berrazaga*, ${ }^{1} \mathrm{~N}$ Mejri, ${ }^{1} \mathrm{H}$ Rachdi, ${ }^{2} \mathrm{M}$ Ferjaoui, ${ }^{1} \mathrm{~N}$ Daoud, ${ }^{1} \mathrm{H}$ Boussen. ${ }^{7}$ Abdrahman Mami hospital medical oncology department Tunisia, Medical Oncology, Ariana, Tunisia; ${ }^{2}$ maternity and neonatal center of Tunis, B, Tunis, Tunisia

\subsection{6/ijgc-2021-IGCS.285}

Objectives Epithelial ovarian cancers relapse in the majority of cases. We aimed to analyze overall survival prognostic factors in recurrent epithelial ovarian cancer in Tunisia.

Methods We conducted a retrospective monocentric study including 60 Tunisian patients with relapsed epithelial ovarian cancer between October 2012 and December 2018. Clinicopathological Data, treatment and survival data were collected from medical records. The Kaplan-Meier method was used to calculate overall survival (OS) and Cox regression analysis was performed to define the effects of risk factors on survival.

Results The median age at diagnosis was 59 years [28-85 years]. Recurrent ovarian cancer were platinum sensitive in $35 \%(n=21)$, partially sensitive in $30 \%(n=18)$ and platinum-resistant in $35 \%(n=21)$ of cases. Surgery of the first recurrence was performed for 9 patients (15\%). Fifty-three $(88 \%)$ patients received at least one line of chemotherapy in the recurrence setting. The median number of received cycles was one [0-6]. Overall survivals at 1 year, 2 years and 5 years were respectively $83,3 \%, 62,1 \%$, and $37,3 \%$. Median overall survival was 32 months. Prognostic factors which were associated with better OS were early initial stage (I-IIA) $(p=0.012)$, complete initial staging $(p=0.001)$, platinum- sensitive relapse $(p=0.001)$ and $R 0$ resection of the relapse $(p=0.001)$. Age, grade, relapse's site didn't impact significantly overall survival.

Conclusions Overall survival of recurrent ovarian cancer remains poor. Further studies in order to promote early detection are needed. Quality of surgery constitutes a major impact factor.

\section{EPV215/\#519 SOX4 DRIVES OVARIAN CANCER STEMNESS VIA TRANSCRIPTIONALLY ACTIVATING HDAC1}

1,2,3 Liu $^{*},{ }^{1,2,3}$ T Lang, ${ }^{2} \mathrm{D}$ Zou, ${ }^{2} \mathrm{D}$ Wang, ${ }^{2} Y$ Tang, ${ }^{2} \mathrm{R}$ Li, ${ }^{1,2,3} \mathrm{Y} \mathrm{Li},{ }^{1,2} \mathrm{D}$ Ding, ${ }^{1,2,3} \mathrm{Q}$ Zhou. ${ }^{1}$ Chongqing University, College of Bioengineering, Chongqing, China; ${ }^{2}$ Chongqing University Cancer Hospital, Department of Gynecologic Oncology, Chongqing, China; ${ }^{3}$ Chongqing University Cancer Hospital, Chongqing Key Laboratory of Translational Research for Cancer Metastasis and Individualized Treatment, Chongqing, China

\subsection{6/ijgc-2021-IGCS.286}

Objectives The objective of this study is to explore the role of SOX4 in ovarian cancer stem cells (OCSCs) and elucidate the underlying mechanisms.

Methods Ovarian cancer cell lines and primary cells were used in this study. Lentivirus system was used for genetic manipulation. Sphere-forming activity was examined by sphere formation assay. limiting dilution assay was employed to determine the frequency of sphere-forming cell. The mRNA and protein levels were determined by qRT-PCR and western blot, respectively. The effect of SOX4 on the transcriptional activity of HDAC1 promoter was tested by luciferase assay and their direct binding was determined by Chromatin immunoprecipitation (ChIP) assay. The protein levels of surface markers were examined by flowcytometry.

Results SOX4 overexpression significantly increased the sphere-forming activities, the frequency of sphere-forming cells and the expression levels of OCSCs markers in OCSCs; SOX4 depletion led to opposite results. SOX4 directly bound to the HDAC1 promoter and increased the transcriptional activities of HDAC1 promoter and there are four SOX4-bidning sites in HDAC1 promotor. HDAC1 predicts poor prognosis of ovarian cancer and positively regulates OCSCs stemness. HDAC1 ablation abolished the effect of SOX4 on OCSCs stemness.

Conclusions The results in this study demonstrated a novel mechanism that SOX4 promotes ovarian cancer stemness by transcriptionally activating HDAC1. This finding suggests that HDAC1 inhibitor could be an effective therapeutic agent for eradicating human OCSCs driven by aberrant SOX4 upregulation.

\section{EPV216/\#520 PARP1 SUPPORTS OVARIAN CANCER STEMNESS BY UBIQUITINATION OF NUCLEAR YAP}

1,2,3 T Lang ${ }^{*}$, 1,2,3 $\mathrm{LLiu}^{1}{ }^{1} \mathrm{D}$ Zou, ${ }^{1} \mathrm{D}$ Wang, ${ }^{1} Y$ Tang, ${ }^{1} \mathrm{R}$ Li, ${ }^{1,2,3} \mathrm{Y}$ Li, ${ }^{1,3} \mathrm{D}$ Ding, ${ }^{1,2,3} \mathrm{Q}$ Zhou. ${ }^{1}$ Chongqing University Cancer Hospital, Department of Gynecologic Oncology, Chongqing, China; ${ }^{2}$ Chongqing University Cancer Hospital, Chongqing Key Laboratory of Translational Research For Cancer Metastasis and Individualized Treatment, Chongqing, China; ${ }^{3}$ Chongqing University, College of Bioengineering, Chongqing, China

\subsection{6/ijgc-2021-IGCS.287}

Objectives The objective of this study is to improve our understanding about PARP1 in ovarian cancer stem cells (OCSCs), which would be helpful for extension of the potential of PARP inhibitors as anti-cancer drugs.

Methods SK-OV3, primary ovarian cancer cells and xenograft mice were used in the study. Genetic manipulation was performed by lentivirus systems. The stemness of OCSCs was 